\title{
Surgical treatment of secondary hyperparathyroidism in elderly patients: an institutional experience
}

\author{
Andrea Polistena $^{1}$ (D) Alessandro Sanguinetti $^{1} \cdot$ Roberta Lucchini $^{1}$ • \\ Segio Galasse ${ }^{1} \cdot$ Stefano Avenia $^{1} \cdot$ Massimo Monacelli $^{1} \cdot$ Louis Banka Johnson $^{2}$. \\ Bengt Jeppsson ${ }^{2} \cdot$ Nicola Avenia $^{1}$
}

Received: 29 August 2016/Accepted: 1 November 2016/Published online: 9 November 2016

(C) Springer International Publishing Switzerland 2016

\begin{abstract}
Background Secondary hyperparathyroidism in elderly fragile patients presents clinical difficulties due to severity of symptoms and related comorbidity. The optimal surgical approach for this group of patients is still debated.

Aim The aim of the study was to define the optimal technique of parathyroidectomy in elderly patients with secondary hyperparathyroidism.

Methods Retrospective analysis in a series of 253 patients including 35 elderly individuals at a single institution was carried out. Postoperative parathyroid hormone decrease, surgical complications and symptoms control were analyzed for all patients in relation to the types of parathyroidectomy performed.

Results In elderly patients, total parathyroidectomy was the most used approach. Subtotal parathyroidectomy was mostly reserved for younger patients suitable for kidney transplantation. No elderly patients treated with total parathyroidectomy were autotransplanted. No significant difference in surgical complications was observed between younger and elderly patients and considering the different procedures. Adequate symptom control after surgery was achieved in almost $90 \%$ of patients. A limited rate of recurrence requiring repeat surgery was observed only after subtotal parathyroidectomy.
\end{abstract}

Andrea Polistena

apolis74@yahoo.it

1 UOC Chirurgia Generale e Specialità Chirurgiche, Endocrine Surgery Unit, Medical School, S. Maria University Hospital, University of Perugia, via Tristano di Joannuccio 1, 05100 Terni, Italy

2 Surgical Department, Skåne University Hospital, University of Lund, Malmö, Sweden
Discussion Considering the features of all types of parathyroidectomy, very low recurrence rate, contained postoperative hypocalcemia and limited complications following total parathyroidectomy, might represent specific advantages for elderly patients.

Conclusions Total parathyroidectomy without parathyroid transplantation is safe for elderly patients with secondary hyperparathyroidism and a good alternative to the wellestablished total parathyroidectomy with autografting.

Keywords Secondary hyperparathyroidism - Subtotal parathyroidectomy · Total parathyroidectomy · Parathyroid autotransplantation · Elderly

$\begin{array}{ll}\text { Abbreviations } \\ \text { SHPT } & \text { Secondary hyperparathyroidism } \\ \text { PTH } & \text { Parathyroid hormone } \\ \text { MIBI } & \text { Sestamibi scintigraphy } \\ \text { US } & \text { Ultrasound } \\ \text { CT } & \text { Computed tomography } \\ \text { MRI } & \text { Magnetic resonance imaging } \\ \text { SPECT } & \text { Single positron emission computed tomography } \\ \text { iPTH } & \text { Intraoperative PTH } \\ \text { IONM } & \text { Intraoperative nerve monitoring }\end{array}$

\section{Introduction}

Secondary hyperparathyroidism (SHPT) is characterized by elevated levels of parathyroid hormone (PTH) due to parathyroid hyperplasia following chronic kidney disease (CKD). Impaired urinary phosphate excretion and hyperphosphatemia, along with low vitamin D levels occurring in CKD, lead to hypocalcemia and continuous overstimulation of the parathyroid glands that induce diffuse 
hyperplasia and hypersecretion of PTH. SHPT patients present with modifications in mineral metabolism and bone disease which result in increased morbidity and decreased quality of life. There is increasing evidence suggesting that these disorders in mineral and bone metabolism are associated with increased risk of cardiovascular calcification, morbidity and mortality [1]. In the great majority of patients, SHPT can be successfully managed by intensified dialysis or medical treatment with phosphate binders and supplementation of calcium and vitamin $\mathrm{D}$, vitamin $\mathrm{D}$ analogs or calcimimetics. With time there are changes in the expression of calcium and vitamin D receptors promoting nodular hyperplasia and inducing adenomatous changes within the parathyroid glands, thus becoming autonomous and difficult to control through conservative treatment [2]. Kidney transplantation remains the treatment of choice in renal failure and is associated with resolution of many of the endocrine and metabolic complications of hyperparathyroidism. For patients not suitable for kidney transplantation, parathyroidectomy is the most effective treatment for severe SHPT in patients not completely responding to pharmacological therapy [3]. Among the different types of surgical procedures for the treatment of SHPT described in the literature, the optimal approach of choice for elderly patients who are not usually suitable for kidney transplantation still remains controversial $[4,5]$. The role of total parathyroidectomy without autotransplantation has been debated since it potentially carries a serious risk of adynamic bone disease with extra-skeletal calcifications and permanent hypocalcemia that requires long-term calcium and vitamin D supplementation [6].

The aim of the present paper is to analyze the different techniques of treatment of SHPT in elderly fragile patients based on an institutional experience.

\section{Patients and methods}

We retrospectively analyzed elderly patients in a cohort of 253 patients with SHPT, operated by the same surgical team, over a period of 20 years, from 1996 to 2015, at the Units of Endocrine Surgery of two university teaching hospitals (S. Maria Hospital, Terni and S. Maria della Misericordia Hospital, Perugia) of Umbria Region, Italy, which are integrated as referral centers for endocrine surgical diseases. Data were collected from our database and analyzed. Informed consent for the use of clinical data for scientific purpose was obtained from all individual participants included in the study. Preoperative work-up included blood texts, ECG, sestamibi scintigraphy (MIBI) and ultrasound (US) of the neck. Computed tomography (CT), magnetic resonance imaging (MRI) or single positron emission computed tomography (SPECT) were used in a few selected cases when diagnosis with simpler procedures failed to localize the disease. In SHPT, indication for surgery was made according to the severity of the clinical stage and to the current guidelines available [3, 6-8]. Guidelines recommend parathyroidectomy in patients with severe SHPT (persistent high serum level PTH $>500-800 \mathrm{pg} / \mathrm{ml}$ ) associated with hyperphosphatemia (serum Phosphorus $>6.0 \mathrm{mg} / \mathrm{dl}$ ) and/or hypercalcemia (serum Calcium $>10.0 \mathrm{mg} / \mathrm{dl}$ ) refractory to at least 6 months of medical treatment. In patients presenting with clinical symptoms (bone and joint pain, muscle weakness, irritability, pruritus, osteoporosis, pathologic bone fracture, anemia, dilated cardiomyopathy, calciphylaxis), surgery is strongly indicated. Also ultrasound detection of an enlarged parathyroid gland (with an estimated volume $>500 \mathrm{~mm}^{3}$ or diameter $>1 \mathrm{~cm}$ ) supports indication for surgery. In the observed population, surgeries performed were subtotal parathyroidectomy, total parathyroidectomy with or without autotransplantation. In all cases, a bilateral exploration was performed. The intraoperative dosage of PTH (iPTH) has been routinely used in the last 10 years. In a limited number of patients during the years 2012 and 2014-2015, the intraoperative nerve monitoring (IONM) with NIM $3.0^{\circledR}$ (Medtronic Italia SpA., Milan, Italy) was used. In all patients, plasma calcium level was tested twice in first postoperative day and once on the second postoperative day, while in hypocalcemic patients daily testing was done until normalization of values. PTH was tested in all patients in first postoperative day and during the nephrological follow-up. Postoperative hypoparathyroidism was defined as a concentration of PTH under $10 \mathrm{ng} / \mathrm{L}$ (normal range 10-65 ng/ L) strictly correlated with hypocalcemia defined as an ionized calcium level (iCa) under $1.0 \mathrm{mmol} / \mathrm{L}$ (normal range $1.12-1.32 \mathrm{mmol} / \mathrm{L}$ ). Treatment of hypoparathyroidism was carried out as previously described [9]. Type of the procedure, surgical complications (recurrent laryngeal nerve lesion, severe hypoparathyroidism), hospital stay, postoperative PTH and calcium/phosphorum values and symptoms control (bone and joint pain, muscle weakness, irritability, pruritus, osteoporosis, pathologic bone fracture, anemia, dilated cardiomyopathy, calciphylaxis) were considered for outcome. Surgical follow-up was regularly carried out in the outpatient clinic for at least 12 months.

\section{Statistical analysis}

We used Student's t test for analysis of variance between groups when applicable. A $p$ value $<0.05$ was considered statistically significant. All of the data were analyzed using XLSTAT (Addinsoft, New York, NY, USA). 


\section{Results}

The cohort of 253 patients who underwent surgery for SHPT was divided into two groups: 218 patients younger than 65 years (group A) and 35 (13\%) elderly patients older than 65 years (group B). Surgery performed is presented in Table 1. Subtotal parathyroidectomy was performed in 122 patients of group A and in 7 of group B. Total parathyroidectomy with autotransplantation was performed exclusively in patients of group A (15 cases). Total parathyroidectomy alone was performed in 88 and 28 patients, respectively, of group A and B. We either observed recurrent or persistent SHPT following subtotal parathyroidectomy in 19 patients, all in group A, who were successfully reoperated within 6 months of surgery with total parathyroidectomy. Thymectomy was added to total parathyroidectomy only in those cases in which a persistent high iPTH was observed during surgery or when preoperative imaging showed mediastinal glands or glandular activity or at reoperation for recurrent or persistent SHPT. In $96 \%$ of cases, patients were discharged on the second postoperative day, after monitoring of postoperative calcium and PTH. Unilateral temporary recurrent laryngeal nerve palsy was observed in two cases $(0.9 \%)$ in group $\mathrm{A}$, one following total and the other following subtotal parathyroidectomy. The only case $(0.4 \%)$ of unilateral permanent palsy was observed in group $\mathrm{A}$, following total parathyroidectomy. Postoperative PTH and calcium/phosphorus values returned to normal or acceptable values in 92.4\% of patients at one-month follow-up with no need for reoperation. A limited number of patients needed long-term calcium/vitamin D support after total parathyroidectomy (25 patients in group A and 8 in group B, respectively). Symptom control was achieved in $90 \%$ of patients with no significant differences comparing the different procedures. All the patients at one-year follow-up were doing well with a general improvement in their quality of life.

\section{Discussion}

Patients with severe SHPT suffer from several clinical symptoms such as bone and joint pain, muscle weakness, irritability, bone loss, anemia resistant to erythropoietin, cardiomyopathy, calciphylaxis. Although the majority of patients with SHPT are initially managed by medical treatment, this approach does not always provide adequate control of symptoms. The evolution of the disease usually requires a surgical approach, including parathyroidectomy and percutaneous ethanol injection. Successful surgical treatment usually achieves a drop in PTH levels, relief from some clinical symptoms and a reduction in mortality [10-15]. The surgical procedures available for the treatment of SHPT with diffuse incretion of PTH are subtotal parathyroidectomy, total parathyroidectomy with autotransplantation and total parathyroidectomy without autotransplantation. Every procedure has its own disadvantages, and it is still unclear which approach is best. Furthermore, it is not completely clear which type of surgery is safest for the elderly fragile patients affected by the disease.

Subtotal parathyroidectomy was first described in 1960 Sanbury [16]. The parathyroid gland to be preserved is usually that in a favorable position together with the recurrent laryngeal nerve, weighing less than $1 \mathrm{~g}$ and smaller than $1 \mathrm{~cm}$. Only one-third of the gland has to be left in place and is marked with 5/0 not absorbable suture with titanium clips applied to it. The major limitation of subtotal parathyroidectomy is that it is very difficult to decide which gland to be chosen for preservation by simple visual evaluation only. This situation is the principle limiting factor of the procedure and is the main cause of recurrent hyperparathyroidism after surgery. Once recurrence occurs, re-exploration of the neck is then indicated but this is associated with a significantly higher risk of injury to the recurrent laryngeal nerves than at initial surgery. In redo surgery, risk of parathyromatosis is increased [17].

In our series, the cases of recurrent SHPT were observed in group A, primarily after subtotal parathyroidectomy. In elderly fragile patients, reoperation is associated with increased morbidity and eventually also mortality although it is relatively rare. Control of the serum calcium levels may be difficult to monitor after kidney transplantation. Young patients who are on a waiting list for kidney transplantation have to be offered primarily subtotal parathyroidectomy, which results in reduced parathyroid

Table 1 Types of procedures carried out in group A and B

\begin{tabular}{lcc}
\hline Operation & Group A (<65 years) & Group B (>65 years) \\
\hline Subtotal parathyroidectomy (n) & 115 & 7 \\
Total parathyroidectomy + parathyroid autotransplantation (n) & 15 & 0 \\
Total parathyroidectomy (n) & 88 & 28 \\
Total (n) & 218 & 35 \\
Reoperation for persistent or recurrent SHPT (n) & 19 & 0 \\
\hline
\end{tabular}


activity with lower risk of post-transplantation hypocalcemia [18]. Elderly patients are usually not offered transplantation. This approach is associated with a significant risk of recurrence with consequent increased morbidity which offers no advantages, in terms of limiting hypocalcemia in these fragile patients as it does in the transplanted ones. Therefore, subtotal parathyroidectomy might not represent the standard indication for this category of SHPT patients, and according to this rationale in our experience, only $7(6 \%)$ out of 122 patients operated with this approach were allocated in group B.

Total parathyroidectomy and autotransplantation were first performed by Wells in 1975 [19]. In this technique, a small portion of resected parathyroid tissue is transplanted in the forearm in non dominant limb or in the opposite side of the arteriovenous fistula, in the fibers of the brachioradialis muscle or alternatively in the neck, in the sternocleidomastoid muscle or subcutaneously. The rationale of the procedure is to achieve a complete elimination of the disease in the neck and to contain the hypoparathyroidism occurring after total parathyroidectomy alone. If a recurrent SHPT should develop, it is easier and safer to remove the residual parathyroid tissue from the forearm compared to neck re-exploration that is associated with increased morbidity. Indication for this approach at surgery might be due to the presence of four large glands, suggesting more of a nodular than diffuse hyperplasia, with consequent higher risk of neck recurrence if they should undergo subtotal resection. With recurrence ranging from 5 to $80 \%$ following total parathyroidectomy with autotransplantation, as similarly reported for subtotal parathyroidectomy $[7,20]$, the choice of an extra-cervical site, where reoperation could be easier and safer, is therefore recommended. Probably due to limited use of total parathyroidectomy with autotransplantation in our series, we did not experience cases of recurrence after surgery with this approach. A potential disadvantage of the procedure is the difficulty, in case of recurrence, of defining whether it is due to the transplanted tissue or to an ectopic residual gland, whose removal is anyway difficult $[20,21]$. Although malignant transformation of the graft is extremely rare, its occurrence is always possible and it might require a demanding procedure for radical surgery with cases of severe calciphylaxis being reported [7]. The alternative option of subcutaneous parathyroid autografting may be advisable because if reoperation is required, the procedure is simple and reliable and all or a portion of the graft may be removed under local anesthesia and in case of malignancy with severe calciphylaxis a radical excisions may be performed without sacrificing vital structures [7]. Other authors support the use of the sternocleidomastoid muscle as a better site for autografting, advocating the same rationale of easy accessibility, one operative site, less graft ischemia, low incidence of infection and high success rate due to excellent blood supply. This specific advantage is observed in patients with bilateral artificial arteriovenous fistula for hemodialysis, which excludes the use of the forearm for the grafting. Similarly if a recurrence develops, the classical imaging modality would be able to distinguish between a cervical recurrence and a graft depending on recurrence [14]. Furthermore, the autograft initially is not promptly active, and this may be associated with longer postoperative hypocalcemia than following subtotal resection. After all types of parathyroidectomy, the lack of osteoclastic activity caused by a decrease in PTH postoperatively may lead to a precipitous fall in calcium levels, a condition called hungry bone syndrome. Since the autographed parathyroid tissue begins to function two to three weeks after surgery, patients may have severe hungry bone syndrome in this period and may therefore need to receive calcium and calcitriol supplement [22].

According to the current guidelines, total parathyroidectomy with forearm autograft is mainly recommended in patients with long-term hemodialysis after surgery and not candidates for kidney transplantation [6, 17].

This approach is definitely well standardized but is associated, as above described with risk of recurrence and of potential temporary hypocalcemia. Although this is not the first choice in our institution, it certainly could represent a valid option even in elderly patients.

Total parathyroidectomy without autotransplantation is a third valid approach available in SHPT patients mainly aiming to avoid recurrences. According to the rationale of total parathyroidectomy, all parathyroid glands including supernumerary and ectopical ones ought to be removed at the initial operation. Nevertheless, even after accurate bilateral exploration an unknown ectopic gland or a very small one may rarely be missed [7, 23].

Total parathyroidectomy has recently received increasing interest since several authors demonstrate low recurrence rates without evidence of clinical bone disease. Total parathyroidectomy has the one clear advantage that an almost inexistent risk of recurrence noted between 0 and $4 \%$ compared to $5-80 \%$ which is observed following subtotal or total parathyroidectomy with parathyroid autografting [2]. The potential disadvantage of this approach is that it carries a certain risk of adynamic bone disease and permanent hypocalcemia requiring long-term calcium and vitamin D supplementation [6], but in our series this was observed only in limited cases.

However, even when all parathyroid glands are removed, PTH levels are usually measurable after surgery because nets of parathyroid cells remain frequently localized in the thyroid and mediastinum [7]. This evidence supports the recommendation of the mediastinal exploration and thymectomy to resect additional parathyroid 
glands, optimizing the reduction in PTH and the normalization of calcium/phosphorus homeostasis when doing total parathyroidectomy. The excision of the retromanubrial fibrous fatty tissue including thyrothymic ligament might be therefore recommended during parathyroidectomy, but it is not routinely performed [7, 24, 25].

As in our series, specific indication for total parathyroidectomy might be referred for elderly patients who are not suitable for kidney transplantation and severe calciphylaxis. The clear advantages of the procedure are represented by very low rate of recurrence and optimal control of calciphylaxis. This result as observed in our series is gained with no significant increase in morbidity although the major bias of our study is the limited number of elderly patients enrolled.

The preoperative parathyroid localization study is currently adopted in surgery for primary hyperparathyroidism. However, its routine use is controversial in surgery for SHPT due to the need for a bilateral neck exploration to identify all glands as is mandatory in all cases. Nevertheless, in SHPT the localization of abnormal orthotopic and ectopic glands might be a useful tool to prevent recurrent or persistent disease and to perform a safer and effective surgery. Some authors report a clear advantage of this method in their series [26]. The effect of preoperative imaging localization on the surgical outcomes was investigated by measuring the iPTH reduction for positive or negative imaging localization. This was greater in positive than negative imaging localization although not significant [26]. The study showed that US had the highest sensitivity (91.5\%) and MIBI had the lowest $(56.1 \%)$ after CT scan. It was also observed that the preoperative imaging localization did not affect the recurrence or persistence of SHPT. For this reason, imaging techniques might be more indicated for redo surgery when ectopic or supernumerary glands cannot be identified or when recurrent disease occurs, despite adequate first-time surgical exploration [26].

In total parathyroidectomy with or without autotransplantation, all parathyroid glands must be removed completely from the neck during surgery for a successful outcome but this is affected by the risk of supernumerary parathyroid glands [27].

Similarly, since in patients with SHPT, bilateral exploration and total gland excision is needed, the value of iPTH is less important as the need for preoperative localization. However, some studies suggest that iPTH improves surgical results, especially in patients with three or five glands and in patients with ectopic parathyroid tissue. Therefore, the iPTH might be a useful diagnostic tool during parathyroid surgery for prediction of postoperative outcome and thereby impacts on the decision for further exploration or not [28-31]. The predictive value of intraoperative and perioperative PTH was shown to improve not only the success rate of operation but also to reduce the complications caused by unnecessary exploration. Furthermore, perioperative iPTH monitoring could help in detecting persistent SHPT in an early stage after surgery and allows a correct schedule for medical treatments or reoperation also in the elderly population [32-37].

Since adoption of iPTH in combination with imaging localization in the surgical treatment of SHPT at our units in the last ten years, we have found it a useful tool during parathyroidectomy. However, we have no specific data to support improvement in surgical results following parathyroidectomy with iPTH measurement.

\section{Conclusions}

Total parathyroidectomy with parathyroid tissue autotransplantation is a feasible, safe and effective surgical option for the patients with SHPT. Nevertheless, for elderly patients total parathyroidectomy without autografting might be a better option due to very low recurrence rates, contained hypocalcemia without severe bone disease and limited complications. Since elderly patients are not fit for kidney transplantation, we consider subtotal parathyroidectomy not a good surgical approach for this group of patients. The adoption of adequate preoperative imaging study and use of iPTH dosage might be useful tools during parathyroidectomy. Further randomized studies are, however, required to shed more light on the surgical management of the fragile elderly patients.

\section{Compliance with ethical standards}

Conflict of interest The authors declare that they have no conflict of interest.

Ethical approval For this type of study, formal consent is not required.

Informed consent Informed consent was obtained from all individual participants included in the study.

\section{References}

1. Block GA, Cunningham J (2006) Morbidity and mortality associated with abnormalities in bone and mineral metabolism in CKD. In: Olgaard K (ed) clinical guide to the basics of bone and mineral metabolism in CKD. National Kidney Foundation, New York, pp 77-92

2. Bratucu MN, Garofil ND, Radu PA et al (2015) Measurement of quality of life after total parathyroidectomy in patients with secondary hyperparathyroidism and end stage renal disease. Chirurgia (Bucur) 110:511-517

3. Kidney Disease: Improving Global Outcomes (KDIGO) CKDMBD Work Group (2009) Clinical practice guideline for the 
diagnosis, evaluation, prevention, and treatment of chronic kidney disease-mineral and bone disorder (CKD-MBD). Kidney Int 188:S1-S130

4. Drakopoulos S, Koukoulaki M, Apostolou T et al (2009) Total parathyroidectomy without autotransplantation in dialysis patients and renal transplant recipients, long-term follow-up evaluation. Am J Surg 198:178-183

5. Iwamoto N, Sato N, Nishida M et al (2012) Total parathyroidectomy improves survival of hemodialysis patients with secondary hyperparathyroidism. J Nephrol 25:755-763

6. Messa P (2003) Italian society of nephrology, [Renal osteodystrophy Guidelines]. G Ital Nefrol 24:S83-S95

7. Ockert S, Willeke F, Richter A et al (2002) Total parathyroidectomy without autotransplantation as a standard procedure in the treatment of secondary hyperparathyroidism. Langenbecks Arch Surg 387:204-209

8. Guideline Working Group, Japanese Society for Dialysis Therapy (2008) Clinical practice guideline for the management of secondary hyperparathyroidism in chronic dialysis patients. Ther Apher Dial 12:514-525

9. Polistena A, Monacelli M, Lucchini R et al (2015) Surgical morbidity of cervical lymphadenectomy for thyroid cancer: a retrospective cohort study over 25 years. Int J Surg 21:128-134

10. Gallieni M, Corsi C, Brancaccio D (2000) Hyperparathyroidism and anemia in renal failure. Am J Nephrol 20:89-96

11. Goto N, Tominaga Y, Matsuoka S et al (2005) Cardiovascular complications caused advanced secondary hyperparathyroidism in chronic dialysis patients; specific focus on dilated cardiomyopathy. Clin Exp Nephrol 9:138-141

12. Richards ML, Wormuth J, Bingener J et al (2006) Parathyroidectomy in secondary hyperparathyroidism: is there optimal operative management? Surgery 139:174-180

13. Yajima A, Ogawa Y, Takahashi HF et al (2003) Changes of bone remodeling immediately after parathyroidectomy for secondary hyperparathyroidism. Am J Kidney Dis 42:729-738

14. Tominaga Y, Uchida K, Haba T et al (2001) More than 1000 cases of total parathyroidectomy with forearm autograft for renal hyperparathyroidism. Am J Kidney Dis 38:S168-S171

15. Kestenbaum B, Andress DL, Schwartz SM et al (2004) Survival following parathyroidectomy among United States dialysis patients. Kidney Int 66:2010-2016

16. Sanbury SW, Lumb GA, Nicholson WF (1960) Elective subtotal parathyroidectomy for renal hyperparathyroidism. Lancet 1:793-799

17. Gasparri G, Ferrarotti G, Natì L (2008) Iperparatiroidismo secondario. In: Rosato L (ed) Trattato Italiano di endocrinochirurgia. Club delle U.E.C. Grafica Santhiatese, Italy, pp 487-491 (ISBN: 978-88-87374-98-8)

18. Lou I, Foley D, Odorico SK et al (2015) How well does renal transplantation cure hyperparathyroidism? Ann Surg 262:653-659

19. Wells SA Jr, Gunnells JC, Shelburne JD et al (1975) Transplantation of the parathyroid glands in man: clinical indications and results. Surgery 78:34-44

20. Puccini M, Carpi A, Cupisti A et al (2010) Total parathyroidectomy without autotransplantation for the treatment of secondary hyperparathyroidism associated with chronic kidney disease: clinical and laboratory long-term follow-up. Biomed Pharmacother 64:359-362

21. Agha A, Loss M, Schlitt HJ et al (2012) Recurrence of secondary hyperparathyroidism in patients after total parathyroidectomy with autotransplantation: technical and therapeutic aspects. Eur Arch Otorhinolaryngol 269:1519-1525

22. Goldfarb M, Gondek SS, Lim SM et al (2012) Postoperative hungry bone syndrome in patients with secondary hyperparathyroidism of renal origin. World J Surg 36:1314-1319

23. Conzo G, Perna A, Candela G et al (2012) Long-term outcomes following "presumed" total parathyroidectomy for secondary hyperparathyroidism of chronic kidney disease. G Chir 33:379-382

24. Diaconescu M, Glod M, Costea I et al (2014) Thyroid and thymic exeresis in surgery of hyperparathyroidism. Chirurgia 109:747-752

25. Sakman G, Parsak CK, Balal M et al (2014) Outcomes of total parathyroidectomy with autotransplantation versus subtotal parathyroidectomy with routine addition of thymectomy to both groups: single center experience of secondary hyperparathyroidism. Balk Med J 31:77-78

26. Lee JB, Kim WY, Lee YM (2015) The role of preoperative ultrasonography, computed tomography, and sestamibi scintigraphy localization in secondary hyperparathyroidism. Ann Surg Treat Res 89:300-305

27. Schneider R, Waldmann J, Ramaswamy A et al (2011) Frequency of ectopic and supernumerary intrathymic parathyroid glands in patients with renal hyperparathyroidism: analysis of 461 patients undergoing initial parathyroidectomy with bilateral cervical thymectomy. World J Surg 35:1260-1265

28. Echenique Elizondo M, Diaz-Aguirregoitia FJ, Amondarain JA et al (2005) Intraoperative monitoring of intact PTH in surgery for renal hyperparathyroidism as an indicator of complete parathyroid removal. World J Surg 29:1504-1509

29. Seehofer D, Rayes N, Klupp J et al (2005) Predictive value of intact parathyroid hormone measurement during surgery for renal hyperparathyroidism. Langenbecks Arch Surg 390:222-229

30. Lokey J, Pattou F, Mondragon-Sanchez A et al (2000) Intraoperative decay profile of intact (1-84) parathyroid hormone in surgery for renal hyperparathyroidism: a consecutive series of 80 patients. Surgery 128:1029-1034

31. Barczynski M, Cichon S, Konturek A et al (2005) A randomised study on a new cost-effective algorithm of quick intraoperative intact parathyroid hormone assay in secondary hyperparathyroidism. Langenbecks Arch Surg 390:121-127

32. Zhang L, Xing C, Shen C et al (2016) Diagnostic accuracy study of intraoperative and perioperative serum intact pth level for successful parathyroidectomy in 501 secondary hyperparathyroidism patients. Sci Rep 6:26841

33. Gervasi R, Orlando G, Lerose MA et al (2012) Thyroid surgery in geriatric patients: a literature review. BMC Surg 12:S16

34. Amato B, Compagna R, Sivero L et al (2013) Lymphectomy for elderly in thyroid surgery. Chirurgia 26:303-306

35. Desiato V, Melis M, Amato B et al (2016) Minimally invasive radioguided parathyroid surgery: a literature review. Int J Surg 28:S84-S93

36. Rispoli C, Rocco N, Iannone L et al (2009) Developing guidelines in geriatric surgery: role of the grade system. BMC Geriatr 9:A99

37. Kim WY, Lee JB, Kim HY (2012) Efficacy of intraoperative parathyroid hormone monitoring to predict success of parathyroidectomy for secondary hyperparathyroidism. J Korean Surg Soc 83:1-6 